\title{
Two-dimensional simulations of laser-plasma interaction and hot electron generation in the context of shock-ignition research
}

\author{
O. Klimo $^{1}$, J. Psikal ${ }^{1}$, V. T. Tikhonchuk ${ }^{2}$, S. Weber ${ }^{3}$ \\ ${ }^{1}$ Czech Technical University in Prague, Faculty of Nuclear Sciences and Physical \\ Engineering, Brehova 7, 11519 Prague, Czech Republic \\ ${ }^{2}$ Univ. Bordeaux - CEA - CNRS, Centre Lasers Intenses et Applications, UMR 5107, \\ 33405 Talence Cedex, France \\ ${ }^{3}$ ELI-Beamlines project, Institute of Physics, ASCR v.v.i., Na Slovance 2, 18221 \\ Prague, Czech Republic \\ E-mail: ondrej.klimo@fjfi.cvut.cz
}

\begin{abstract}
Laser plasma interaction and hot electron generation play crucial role in the context of inertial confinement fusion and in particular in the shock ignition concept. We present here fully kinetic large-scale two-dimensional simulation studying laser plasma interaction and hot electron generation in a relatively long and hot coronal plasma. The simulation shows saturation of the reflectivity of an intense spike pulse and absorption taking place close to the quarter critical density in particular due to cavitation and stimulated Raman scattering. Signatures of steady two-plasmon decay are observed, but the hot electron number produced by this instability is low in comparison with the other two processes. The spectral and angular distribution of the back-scattered light is presented and the energy and angular characteristics of hot electrons due to individual absorption processes are studied.
\end{abstract}

PACS numbers: $52.35 . \mathrm{Mw}, 52.38 . \mathrm{Bv}, 52.38 . \mathrm{Kd}, 52.65 . \mathrm{Rr}$

Submitted to: Plasma Phys. Control. Fusion 


\section{Introduction}

Ignition of a precompressed fusion pellet can be achieved by using a strong shock wave $[1,2]$. This concept of Inertial Confinement Fusion (ICF) is called Shock Ignition (SI). The shock wave has to raise the pressure in the target hot spot to the order of $0.5 \mathrm{Tbar}$ (for the standard hot spots radius of $20-30 \mu \mathrm{m}$ ). About 200-300 TW of laser power have to be absorbed to create sufficient pressure in the ablation layer to launch the strong shock wave. The nonlinear collective processes are playing an important role in the laser plasma interaction physics in this context. These processes are currently investigated in experiments in several laboratories $[3,4,5,6,7,8,9]$.

Among the nonlinear collective processes, Stimulated Brillouin and Raman Scattering (SBS and SRS, respectively), Filamentation instability (FI) and TwoPlasmon Decay (TPD) are widely studied. These processes are considered as detrimental for ignition for two reasons. The first reason is that they may scatter or reflect significant part of laser pulse energy thus reducing laser absorption. Usually, SBS is considered as the most dangerous one in this respect as it has a high gain and it has been identified as a source of significant reflectivity in many ICF relevant experiments. The second reason for avoiding nonlinear processes is the production of hot electrons due to electron plasma waves excited by these instabilities. Hot electrons may penetrate into the dense shell and deposit there their energy, thus inhibiting compression. It has been demonstrated that electrons with energy below about $100 \mathrm{keV}$ may be stopped in the dense shell surrounding the hot spot in the late phase of compression, when the intense spike of the laser pulse reaches the target [10]. These "not-too-hot" electrons may partially contribute to drive the shock wave as demonstrated in [11]. The electrons produced by TPD have higher temperature than electrons produces by SRS (excluding the forward SRS, which is less likely to play a role) due to smaller wave number and thus higher phase velocity of associated waves. It results that in the case of SI it is desirable to suppress in particular SBS and TPD.

The competition between backward SRS and SBS has been studied in the onedimensional geometry in our previous works $[12,13,14]$ for plasmas with the density scale length at the quarter critical density 150 and $300 \mu \mathrm{m}$, initial electron temperatures about 2 and $5 \mathrm{keV}$ and the laser pulse intensity in the range $1-24 \mathrm{PW} / \mathrm{cm}^{2}$. In these simulations, it has been found that the reflectivity (initially dominated by SBS) stabilises after a transient stage around the value $35 \%$ and SBS is suppressed by density cavities, which develop around the quarter and 1/16th of critical density. The absorbed laser pulse energy goes in particular into hot electrons with the temperature of about $30 \mathrm{keV}$, but the absorption into hot electrons seemes to be overestimated in one-dimensional simulations in comparison with recent experiments [9]. Fully kinetic PIC simulations aiming to study the competition of nonlinear processes in two-dimensional geometry have been performed for a reduced plasma volume in $[16,17]$. They have shown that TPD is excited before SRS and the existence of cavities around quarter critical density in two-dimensional geometry has been confirmed. Formation of cavities was associated 
with the beating of TPD electron plasma waves as this process is excited faster than SRS. The two-dimensional simulations have also demonstrated generation of "not-toohot" electrons, but the boundary conditions (reflecting at the rear side and periodic at the lateral sides) did not allow to follow the interaction on a long time scale and estimate the hot electron temperature and absorption because of hot electron recirculation.

The two-dimensional simulation presented in this paper is performed for similar parameters like in $[16,17]$, but it overcomes the problem of hot electron recirculation by using a special boundary condition absorbing the hot electron flux. This allows us to follow the interaction for a longer time and distinguish hot electron sources. The overall reflectivity of the target for the set of laser and plasma parameters specified below in Sec. 2 is about $36 \%$. This value is similar to the one reported in one-dimensional simulations $[12,13,14]$, but it should not be generalised to other SI configurations. The cavitation at the quarter critical density is also observed and the cavities are identified as responsible for significant absorption into hot electrons with the temperature of about 13 $\mathrm{keV}$. Hot electrons produced by SRS with the temperature of $31 \mathrm{keV}$ are less important, while TPD electrons with temperature $63 \mathrm{keV}$ are negligible as this instability saturates due to cavitation on a rather low and steady level. The hot electrons due to SRS and cavitation propagating into the target have relatively wide angular distribution with full width at half maximum (FWHM) about $72^{\circ}$. The TPD hot electrons propagate out of the target in the direction about $\pm 135^{\circ}$ with respect to laser incidence. Clear density channels in the low density plasma and light filaments are also observed and the maximum light intensity reaches up to 9 times the incident pulse intensity.

This paper is organized as follows. The initial conditions used in the simulation are presented in Sec. 2 together with the simulation model. Sec. 3 contains the results of our simulation and their discussion. In Sec. 3.1, we present the reflectivities and the properties of reflected and transmitted light while in Sec. 3.2, we discuss the properties of hot electrons and the flux of kinetic energy into the target. The most important results are summarized in Sec. 4 together with our concluding remarks.

\section{Simulation model and initial conditions}

The simulation is performed using a massively parallel relativistic electromagnetic PIC code in the two-dimensional geometry. The code was developed from the one previously used in $[12,13,14]$. It uses smooth high order shapes for particles and current assignment using the Zig-Zag algorithm [18], which suppresses numerical heating and allows to reduce the number of particles while keeping a low level of noise. The Maxwell's equations are solved using the standard FDTD scheme on the Yee mesh [19] and the absorbing boundary conditions for fields are done similarly to [20]. The boundary conditions for particles are reflecting on the front side, and thermalizing to the initial temperature on the lateral sides. A special boundary condition to absorb the kinetic energy flux into the target due to hot electrons is used at the rear side similarly like in the one-dimensional simulations [12]. As demonstrated later in Fig. 4 (c), the effect 
of this boundary condition is to keep the energy distribution of particles forming the return current Maxwellian with the initial temperature.

The collisions of plasma particles are not accounted for as the electron-ion collision frequency $\nu_{e i}$ (evaluated at the quarter critical density for the initial electron temperature of $5 \mathrm{keV}$ ) multiplied by the simulation time (assuming the laser wavelength $\lambda=351 \mathrm{~nm})$ is about 1.5. The time between two collisions $\left(1 / \nu_{e i}\right)$ around the quarter critical density is thus much longer than the onset of kinetic processes described in this paper and thus collisions play no role in their saturation. The collisional absorption coefficient calculated for the initial conditions of our simulation is about 0.01 (assuming $\lambda=351 \mathrm{~nm}$ ) and the contribution of collisions to absorption can be neglected. The fact that the simulation is collisionless allows us to rescale the results to another laser wavelength. This may be useful for comparison with some shock ignition experiments performed at different laser wavelength than $351 \mathrm{~nm}[3,6]$.

The simulation cell size is the same in both directions $\Delta x=\Delta y=0.03 \lambda$ and it is similar to the minimum Debye length in the simulation box. The computational time step is about $0.02 \tau$ to fulfill the CFL condition, where $\tau$ is the laser period. The number of (equally weighted) particles per cell is such that each particle (electron or ion) represents $1 \%$ of critical density (e.g. 25 electrons per cell at quarter critical density). The simulation domain contains in total $1.2 \times 10^{8}$ cells and $2.3 \times 10^{8}$ particles. About $2.5 \times 10^{5}$ time steps are performed and the computational time required is about 400000 core CPU hours on the Intel XEON Harpertown CPUs of the JADE cluster of CINES. The simulation time ( $7 \mathrm{ps}$ assuming $\lambda=351 \mathrm{~nm}$ ) is short in comparison with the length of a real ignition laser pulse and it is currently limited by the computational resources. Nevertheless, the time is long enough to study the competition between different nonlinear processes at least in the initial stage of interaction.

The simulation box is about $600 \lambda \times 228 \lambda$. It contains quasineutral deuterium plasma with an exponential density profile and the scale length $L=142 \lambda$. Extensive preparatory one-dimensional simulations confirmed that while the density scale length is about 3 times smaller than in the previous one-dimensional simulations (and in real SI scenario), the dominant physical processes are retained. The minimum plasma density is $1 \%$ and maximum is $40 \%$ of critical density $\left(n_{c}\right)$. The temperatures are constant throughout the target and they are $5 \mathrm{keV}$ for electrons and $1 \mathrm{keV}$ for ions. The target is initially at rest without any macroscopic hydrodynamic motion. The laser pulse is propagating along the $y$ direction and its maximum dimensionless potential $a_{0}$ $\left(I \lambda^{2}=a_{0} \times 1.37 \cdot 10^{18} \mathrm{~W} \mu \mathrm{m}^{2} / \mathrm{cm}^{2}\right.$, where $I$ is the laser pulse intensity) is $a_{0}=0.06$. This corresponds to maximum intensity $40 \mathrm{PW} / \mathrm{cm}^{2}$ for $\lambda=351 \mathrm{~nm}$. Such intensity is higher than the one used in current experiments or the one expected for the spike pulse in SI scheme. Nevertheless, it has been demonstrated in one-dimensional simulations [14] that the laser pulse absorption and hot electron distribution do not significantly change with the laser pulse intensity. The laser pulse has a $430 \tau$ long linear ramp at the beginning and then it stays on the maximum intensity for the rest of the simulation. The transverse laser intensity profile is Gaussian with FWHM $85 \lambda$. This spot size is 
larger than the speckle size in experiments with smoothed laser beams. It corresponds to the size of a tightly focused interaction beam in interaction experiments [6]. It also enables to compare with one-dimensional simulations while retaining the important two-dimensional processes like FI, TPD and side-scattering. The laser pulse is linearly polarized with the electric field component lying in the interaction plane.

\section{Simulation results}

\subsection{Reflected and transmitted light}

The electric and magnetic fields are recorded ten times per period at the front and the rear side of the simulation box (in front of absorbing boundary conditions). These fields are used to find the temporal evolution of reflectivity and transmissivity in the simulation. The Poynting flux component along the laser pulse propagation direction averaged over one laser period and over the transverse size of the simulation box is shown in Fig. 1 (a).
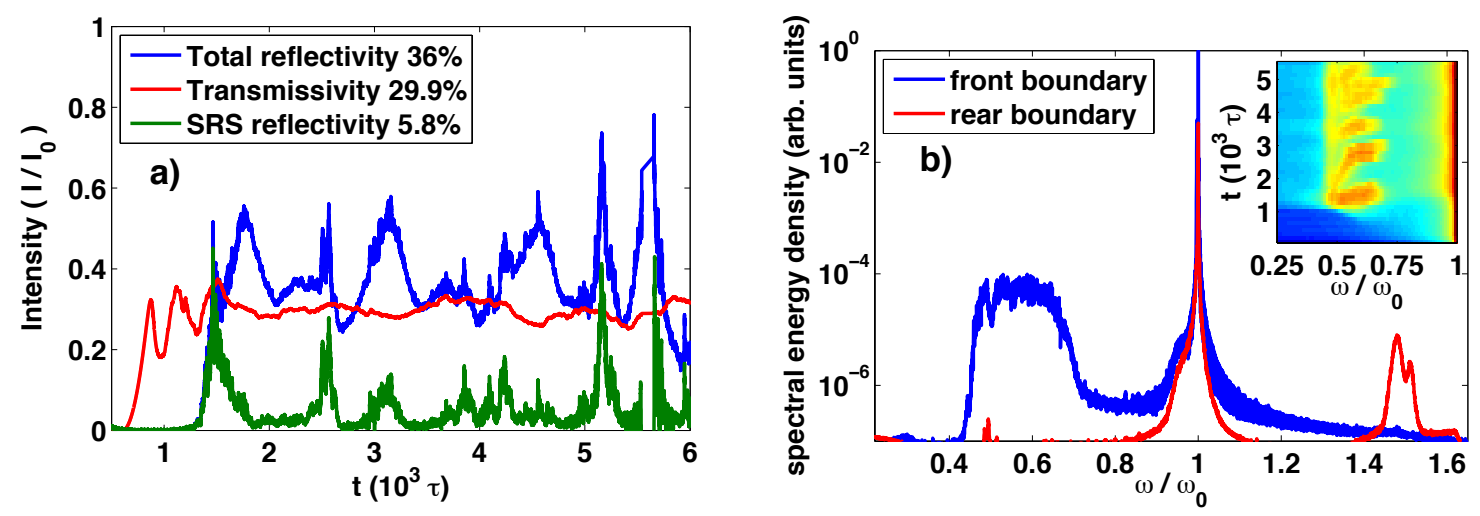

Figure 1. (a) Temporal evolution of the reflectivity and transmissivity in the simulation and the reflectivity in the SRS domain $\left(0-0.9 \omega_{0}\right.$, where $\omega_{0}$ is the laser pulse frequency). The numbers in the legend give the average values during the interaction. Time is measured in thousands of laser periods $(\tau)$. (b) Spectral energy density calculated from the magnetic field perpendicular to the interaction domain at the front and at the rear side of the simulation box. The inset shows the temporal evolution of reflected light spectrum calculated at the front boundary.

The overall temporally averaged reflectivity is about $36 \%$, but the instantaneous one shows intense spikes reaching up to $70 \%$. Moreover, according to Fig. 2 (a), the reflected light intensity can be locally even much higher. Comparing the overall reflectivity with the reflectivity due to SRS only (in the frequency range $0-0.9 \omega_{0}$, where $\omega_{0}$ is the laser pulse frequency), it can be seen that SRS is excited earlier than SBS, but SBS is amplified over a larger region and it dominates the interaction like in the initial transient phase of interaction in one-dimensional simulations [14]. The overall transmissivity of the particular target used in this simulation is stabilized at the level of 
$30 \%$. It demonstrates how much light can propagate through the quarter critical density deeper into the target. The resulting laser absorption in the target is less than $34 \%$ if we don't take into account weak side-scattered radiation, which leaves the simulation box through lateral boundaries. In comparison with one-dimensional simulations [14], the reflectivity saturates at the same level but the absorption is significantly lower.

Figure 1 (b) demonstrates the spectrum of light calculated from the $B_{z}$ field component at the front and the rear side of the target. The signal at the front side of the target combines both, the incident and the backscattered light. Besides the laser pulse itself and the SBS scattered light (both located in a narrow interval around $\omega_{0}$ ), there is quite a broad peak due to SRS with the central frequency $0.58 \omega_{0}$. It comes from plasma with the density in the range $1 / 10$ th to $1 / 4$ th of critical density and it is generated by both, the absolute and the convective SRS. In the temporal evolution of the spectrum shown in the inset of Fig. 1 (b), it is observed that SRS starts at the quarter critical density and it propagates toward the lower density plasma, where it is quenched due to strong Landau damping. The spectrum of transmitted light shows only the transmitted laser light and the signal at $3 / 2 \omega_{0}$, which is a signature of TPD. The signal at $3 / 2 \omega_{0}$ is quite steady during the whole simulation with exception of the initial phase of interaction (where it is higher). The relative importance of TPD in comparison with SRS is estimated below from the electron energy distribution.
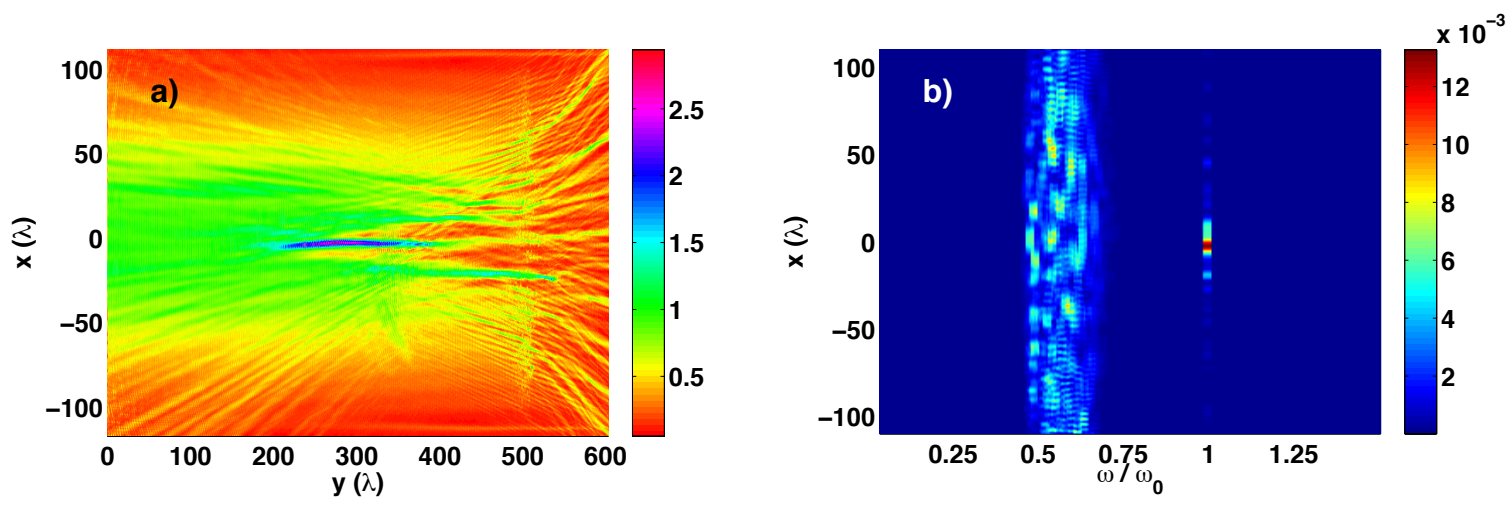

Figure 2. (a) Electromagnetic field amplitude in the simulation box at the time $5300 \tau$. The laser pulse is incident from the left and quarter critical density is at $\sim 500 \lambda$. The units are normalized to the maximum field of the incident laser pulse. (b) The spatially resolved power spectral density of the reflected light recorded at the front boundary and integrated over time 1000-5000 $\tau$. We assume plane waves to be able to distinguish between the incident and the scattered light. The colour scale is linear and the units are arbitrary. The signal in the frequency range $0-0.9 \omega_{0}$ has been multiplied by 20 to fit in the same colour scale like the signal close to $\omega_{0}$.

The snapshot of the electromagnetic field amplitude normalized to the amplitude of the laser field itself is shown in Fig. 2 (a). One can see here a significant amount of light scattered by SBS in one of the filaments. The filamentation of the incident laser beam sets in at the simulation time of about $1400 \tau$ and it is clearly observed behind $y=300 \lambda$ corresponding to the density of about $8 \%$ of the critical density. The 
laser power in this region is already above the threshold for the relativistic self-focusing. The light pulse in Fig. 2 (a) is about $200 \lambda$ long, which is slightly shorter than the length of SBS scattered pulses in one-dimensional simulations [14]. This is perhaps due to shorter density scale length and thus shorter region for the instability amplification. The width of the filament is about $7 \lambda$ and the electromagnetic field intensity is about 9 times higher than that of the incident pulse. This is significant amplification and energy concentration of the incident laser pulse. It is almost 2 times higher than the maximum reflectivity in one-dimensional simulations because the intensity of the SBS scattered pulse is further enhanced by FI. The normalized vector potential of the scattered field in the filament is well below the relativistic potential $\left(a_{0} \simeq 0.18 \ll 1\right)$. However, for higher laser intensity or longer wavelength, the scattered pulse may already accelerate electrons via $\mathbf{v} \times \mathbf{B}$ force.

Most of the light scattered by SBS propagates in a relatively narrow cone of about $4^{\circ}$. This can be estimated from Fig. 2 (b), where the spatially resolved power spectrum density of the scattered light is shown at the front side. In this estimate we further assume the point source of scattered light located about $450 \lambda$ far from the front boundary, which is the point, where most of the SBS scattered light originates. The narrowness of the cone of SBS scattered light justifies our approach that we collect the scattered light only at the front boundary of the simulation domain and neglect scattering on the lateral boundaries. On the other hand, SRS scattering, which is responsible for smaller part of the reflectivity, is not confined to a narrow cone. As we do not have the diagnostic for scattered light at lateral boundaries, we can only give

the minimum value of the width of the angular distribution of SRS scattering. This is estimated in a similar way like for SBS (assuming point source located $450 \lambda$ far from the front boundary) and the minimum value is $27^{\circ}$. The temporal evolution of the electromagnetic field amplitude in the simulation box is shown in the attached movie similarly to Fig. 2 (a). One can see there a very strong interaction at the quarter critical density in the beginning $(\approx 900 \tau)$ and later on strong pulses due the SBS scattering and FI. Weak pulses of back-scattered light propagating with a wide angular distribution before the times 1500, 2600, 3200, 5700 $\tau$ are observed and they correspond to the strong SRS reflectivity in Fig. 1 (a).

The cone, in which the transmitted light propagates, is also relatively broad, about $30^{\circ}$, as can be estimated directly from Fig. 2 (a). The intensity of the transmitted light shows a pattern of small filaments, which are starting in the region around quarter critical density. The pattern changes in time as can be seen in the movie and thus the quarter critical density region works like a dynamic random phase plate as pointed out in $[16]$.

\subsection{Hot electrons}

Our simulation shows approximately round density cavities near the quarter critical density similar as in [17]. Their typical size is of the order of one laser wavelength and 

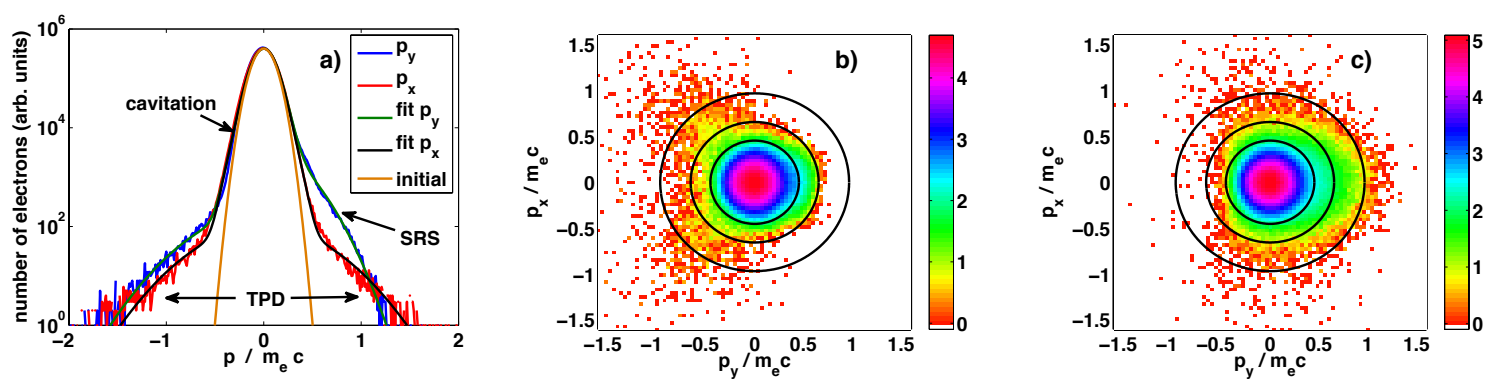

Figure 3. (a) The parallel, $p_{y}$, and the perpendicular, $p_{x}$, components of the momentum distribution of all electrons in the simulation domain $(t=4000 \tau)$. The initial distribution is shown for comparison. The distributions are fitted with three Maxwellian functions with the temperatures 13, 31 and $63 \mathrm{keV}$. The groups of hot electrons with these energies are described in the text. Panels (b) and (c) show the $p_{x} \times p_{y}$ phase space density in the time $6000 \tau$. They are recorded in the region $380 \lambda<y<470 \lambda$ corresponding to the density range $11-23 \% n_{c}$ and the region $470 \lambda<y$ corresponding to density above $23 \% n_{c}$ respectively. The circles correspond to electron kinetic energies 50, 100 and $200 \mathrm{keV}$, the colour scale is logarithmic and the units are arbitrary, but the same in both panels.

they are first induced at about the time of $900 \tau$ by TPD and later by SRS similarly to [16]. Unlike in one-dimensional simulations, the cavities continuously disappear and other cavities are induced. Later in the simulation when the filamentation of the incident laser beam becomes significant at the quarter critical density, the cavities are merged within the light filaments. The cavities together with TPD and SRS are responsible for laser absorption and acceleration of hot electrons. The sources of hot electrons and their properties are analysed below.

Fig. 3 (a) shows the momentum distribution of all electrons in the interaction domain at the time $4000 \tau$ in the direction parallel $\left(p_{y}\right)$ and perpendicular $\left(p_{x}\right)$ to the laser propagation direction. These distributions can be fitted by a sum of several Maxwellian distributions. The initial (isotropic) distribution of electrons is included for comparison. The departure from this distribution shows electron heating in three distinct regions. In the region $p<0.5 m_{e} c$, the heating of electron distribution is almost isotropic. The temperature of these electrons is relatively low and we ascribe them to the cavitation process. The second group of electrons in the region $0.5 m_{e} c<p_{y}<m_{e} c$ is accelerated along the laser propagation direction. They can thus be ascribed to electron plasma waves produced by SRS. The last group of hot electrons forms the hot tail, which is clearly observed in the perpendicular and the backward directions. These electrons are likely accelerated by electron plasma waves induced by TPD. The TPD plasmons have higher phase velocity and wider angular spread compared to SRS.

The distribution function of electrons in the time $6000 \tau$ is shown Fig. 3 (b) and (c). In the lower density part, where the density is below $23 \% n_{c}$, the dominant process producing hot electrons is TPD. These electrons have relatively high energies $(>150$ $\mathrm{keV}$ ), they propagate backward and obliquely with respect to the incident laser pulse. 
The acceleration of hot electrons in the higher density part close to quarter critical density is dominated by SRS. The accelerated electrons have energy mostly below 200 $\mathrm{keV}$ and they propagate in the forward direction into the target.

The angular distribution of electrons is demonstrated in Fig. 4 (a) for the high energy tail. The electrons accelerated by SRS form the forward propagating beam $\left(\theta=0^{\circ}\right)$ and the HWHM (half-width at half-maximum) of the distribution decreases with increasing the minimum energy from $36^{\circ}(E>50 \mathrm{keV})$ to $23^{\circ}(E>150 \mathrm{keV})$. Otherwise, the angular distribution is almost isotropic for lower energy electrons and it is dominated by electrons accelerated in cavities. For energies above $150 \mathrm{keV}$, the electrons produced due to TPD dominate and form a second broad peak in the distribution around the angle $\theta=135^{\circ}$. This is consistent with the momentum distribution in Fig. 3 (a), where the high energy tail has similar temperature for the backward and the transverse part of the distribution and the backward part is having about two times higher amplitude.
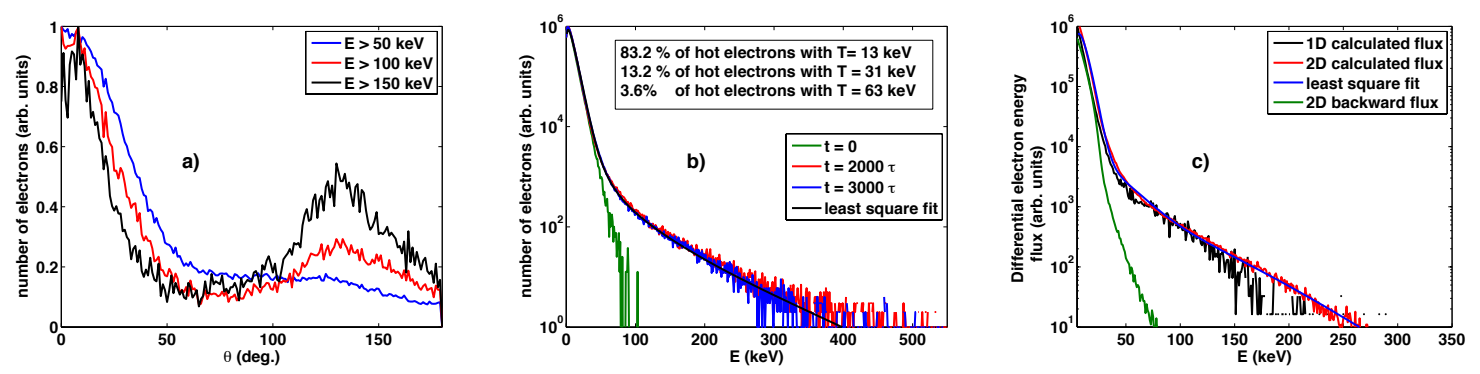

Figure 4. (a) The angular distribution in the simulation box at $6000 \tau$ for electrons with kinetic energy above $50 \mathrm{keV}$ (blue), $100 \mathrm{keV}$ (red) and $150 \mathrm{keV}$ (black). The angle $\theta$ is measured with respect to the laser incidence direction. (b) The electron energy distribution in the whole simulation domain for the time 0,2000 and $3000 \tau$ and the least square fit using a sum of 4 Maxwellian functions to the distribution at $3000 \tau$. The temperatures of hot electrons and the respective fractions of these electrons are in the textbox included in the figure. (c) The differential electron energy flux into the target (in the positive $y$ direction) recorded at the end of simulation and averaged over about $200 \tau$. The flux is recorded in the dense part of the target at the spatial position of about $550 \lambda$. For comparison, we also include a least square fit to the flux assuming an electron distribution with several Maxwellian components as described in the text and a rescaled flux from the 1D simulation with laser pulse intensity $8 \times 10^{15} \mathrm{~W} / \mathrm{cm}^{2}$ published in [14]. The backward flux is included to demonstrate the effect of the absorbing boundary condition.

The energy distributions of all electrons inside the simulation box are shown in Fig. 4 (b). The least square fit using a sum of four Maxwellian distributions to the distribution corresponds to the time $3000 \tau$. The first Maxwellian function describes thermal electrons and the other three components represent hot electrons. The largest number of hot electrons has the temperature of about $13 \mathrm{keV}$, which is in good agreement with the temperature $(10 \mathrm{keV})$ of hot electrons accelerated in cavities in one-dimensional simulations [14]. The second group of hot electrons has the temperature of about 31 
$\mathrm{keV}$. These electrons are accelerated by SRS and their temperature is again in good agreement with one-dimensional simulations [14]. The last group of electrons with the temperature of about $63 \mathrm{keV}$ was not observed in one-dimensional simulations as they originate from TPD. The fractions of hot electrons in the figure indicate that cavitation may be a more important hot electrons source than SRS. This is different from onedimensional simulation results [14].

The instantaneous electron energy distribution cannot give clear answer about the importance of different processes. The hot electrons originate from different locations and they propagate in various directions with the velocity given by their kinetic energy. For example the electrons produced by cavitation have most probably the velocity of about 0.23 velocity of light, while the electrons due to TPD have the velocity more than 2 times higher. If they would have to propagate the same distance before leaving the simulation box through the boundary, the instantaneous electron energy distribution would contain proportionally two times more hot electrons due to cavitation than due to TPD in comparison with the number of hot electrons produced by the source.

To get better understanding of the flux of hot electrons into the target, we record the particles crossing the cells at $y=545 \lambda$, which is located well behind the quarter critical density and close to the rear boundary of the simulation box. This gives us the electron energy flux differential in electron kinetic energy. Provided that the source of hot electrons is quasi-stationary and the electrons do not leave the simulation box through other boundary, this diagnostic describes the source of hot electrons. Hot electrons do not accumulate inside the simulation box but some of them (namely those due to TPD) leave the simulation box through the lateral boundaries. Therefore this diagnostic characterizes the source of hot electrons due to SRS and partially due to cavitation but not due to TPD.

The differential electron energy flux pointing into the target recorded at the end of simulation is plotted in Fig. 4 (c). It is compared with the same flux taken from onedimensional simulation [14] with the laser pulse intensity $8 \mathrm{PW} / \mathrm{cm}^{2}$. Although the laser pulse intensities are different the energy distributions are quite similar in one- and twodimensional simulations. An exception is the higher energy part, where the statistics is very poor in the one-dimensional simulation. The dominant source of hot electrons in the energy range $50-150 \mathrm{keV}$ is SRS in both simulations and the temperature of these electrons is also similar - given by the phase velocity of SRS induced waves close to the quarter critical density. The rescaled flux of electrons is thus similar in this energy range.

The differential electron flux from two-dimensional simulation has been fitted with a sum of several Maxwellian electron distributions. This fit gives temperatures similar to the ones shown in Fig. 4 (b) (namely $12.8 \mathrm{keV}$ and $31.4 \mathrm{keV}$ ) except of the highest temperature component due to TPD. As already explained, the TPD electrons leave the simulation box mostly through the lateral boundaries and they are not recorded in this diagnostic. Integrating the corresponding Maxwellian functions, we are able to find the energy transported into the target by hot electrons components. The result 
is that $78.5 \%$ of hot electron energy is transported by electrons with temperature 12.8 $\mathrm{keV}$ and $21.5 \%$ of energy is transported by hot electrons with the temperature 31.4 $\mathrm{keV}$. To demonstrate the effect of the absorbing boundary condition, we include also the differential electron energy flux of the return current. This flux is similar to the initial one and does not contain any significant high energy tail due to hot electron recirculation.

\section{Conclusions}

We have studied the non-linear processes in laser plasma interaction in the parameter domain relevant to current experiments in the context of shock ignition research using PIC simulations in two-dimensional geometry. The set of initial parameters corresponds to higher laser intensity and smaller plasma scale length due to large computational demands of the simulation. For this set of initial parameters, the overall laser pulse reflectivity is about 36\%, which is quite similar to our previous one-dimensional simulation results [14]. Nevertheless, it is necessary to study more realistic parameters and longer time scales to draw general quantitative conclusions about laser plasma interaction relevant for the shock ignition scenario. Our results instead demonstrate the qualitative behavior of laser plasma interaction that may be expected when the interaction is dominated by parametric instabilities.

The reflectivity in our simulation is dominated by SBS, with the reflected light propagating backward in a narrow cone $\left(4^{\circ}\right)$ and formed by a train of spatially separated intense spikes. SRS emission comes also in a series of bunches, which are separated by significant time delay and the angular distribution of the scattered radiation is much wider (opening angle $>27^{\circ}$ ). Laser pulse absorption is dominated by density cavities, which develop around the quarter critical density. Unlike the case of one-dimensional simulations $[13,14]$, the cavities at $1 / 16$ th of critical density, which may further reduce the SBS reflectivity, are not observed here. It is likely that the simulation time is not

sufficient or that the scattered light in two-dimensional simulations is not intense enough to induce this process.

The cavities play four important roles. They suppress SBS and TPD, they work as a dynamic random phase plate for the transmitted light and they are also a significant source of laser energy absorption. Hot electrons accelerated in the cavities have temperature of about $13 \mathrm{keV}$ and despite of their almost isotropic angular distribution, they are the dominant group of hot electrons transporting the energy deeper into the target. The hot electrons due to SRS have temperature of about $31 \mathrm{keV}$ and originate in the region close to quarter critical density. They propagate into the target in a cone with the opening angle FWHM of $72^{\circ}$. A significant SRS is observed also in a less dense plasma, where it is excited in the inflationary regime. The least important source of hot electrons is due to TPD. The temperature of electrons produced in this process is about $63 \mathrm{keV}$ and these electrons propagate in the backward direction in a cone with a wide opening angle. The electron temperature is in a good agreement with one-dimensional 
simulations $[12,13,14]$ and also with experiments demonstrating hot electrons due to SRS [9] and TPD [7, 15].

\section{Acknowledgments}

The support of O.K. by the Czech Science Foundation, project P205/11/P660 is acknowledged. This work was performed within the framework of the HiPER project EC FP7 \#211737. It is also partly supported by EURATOM within the "Keep-

in-Touch" activities and the Aquitaine Regional Council. The PIC code has been partially modified by J.P. in the frame of the Czech Science Foundation, project P205/12/P366. Support of S.W. by ELI-Beamlines (CZ.1.05/1.1.00/483/02.0061) and OPVK (CZ.1.07/2.3.00/20.0279) projects is acknowledged. The numerical simulations were conducted on the HPC resources of CINES under the allocation 2013-056129 made by GENCI.

\section{References}

[1] Shcherbakov V.A. 1983 Sov. Journ. Plasma Phys. 9240

[2] Betti R. et al. 2007 Phys. Rev. Lett. 98155001

[3] Depierreux S. et al. 2009 Phys. Rev. Lett. 102195005

[4] Baton S.D. et al. 2012 Phys. Rev. Lett. 108195002

[5] Antonelli L. el al. 2011 Acta Tecnica 56 T57

[6] Batani D. et al. 2011 Plasma Phys. Control. Fusion 53124041

[7] Hohenberger M. et al. 2014 Phys. Plasmas 21022702

[8] Theobald W. et al. 2009 Plasma Phys. Control. Fusion 51124052

[9] Theobald W. et al. 2012 Phys. Plasmas 19102706

[10] Betti R. et al. 2008 J. Phys. Conf. Ser. 112022024

[11] Guskov S.Yu. et al. 2012 Phys. Rev. Lett. , 109, 255004

[12] Klimo O. et al. 2010 Plasma Phys. Control. Fusion 52055013

[13] Klimo O. et al. 2011 Phys. Plasmas 18082709

[14] Klimo O. et al. 2013 Plasma Phys. Control. Fusion 55095002

[15] Yaakobi B. et al. 2012 Phys. Plasmas 19012704

[16] Riconda C. et al. 2011 Phys. Plasmas 18092701

[17] Weber S. et al. 2012 Phys. Rev. E 85016403

[18] Umeda T. et al. 2003 Comput. Phys. Commun. 15673

[19] Yee K. 1966 IEEE T. Antenn. Propag. 14302

[20] Umeda T. et al. 2001 Comput. Phys. Commun. 137286 